\title{
The Shortest Route To the Heart
}

\author{
Osama Elshazali ${ }^{1}$, Hafiz Elshazali ${ }^{2}$, and Murtada Ibrahim ${ }^{1}$ \\ ${ }^{1}$ University of Khartoum Faculty of Medicine \\ ${ }^{2}$ North Cumbria University Hospitals NHS Trust
}

January 23, 2022

\begin{abstract}
A six-year-old girl, living in a rural part of Sudan, suffered an accidental penetrating wound to the chest, chest X Ray showed the needle to be embedded within the heart.

The Shortest Route To the Heart

Hafiz Elshazali ${ }^{1}$, Murtada Ibrahim ${ }^{2}$, Osama Elshazali ${ }^{3}$

1. Trust Doctor, North Cumbria Hospital, Whitehaven, UK

2. Paediatric Cardiothoracic Surgeon, University of Khartoum, Sudan

3. Paediatric Cardiologist, University of Khartoum, Sudan

Written informed consent was obtained from the child father to publish this report in accordance with the journal's patient consent policy

Abstract

A six-year-old girl, living in a rural part of Sudan, suffered an accidental penetrating wound to the chest, caused by a sewing needle inserted during a collision with her nine years old sister. She was taken to the nearest district unit, where they tried to remove it but were unsuccessful. She was then transferred to the regional hospital where a chest $\mathrm{X}$ Ray showed the needle to be embedded within the heart
\end{abstract}

(Figure 1). She was referred to the cardiothoracic unit in Khartoum.

She arrived into the cardiothoracic unit 13 days after the incident, on arrival she was haemodynamically stable. Echocardiography showed the needle to be embedded within the myocardium, with a small part protruding into the LV cavity about three millimetre, and a small protrusion into the pericardial space, there was a small rim of pericardial effusion, which did not look haemorrhagic ( Figure 2).

She underwent successful surgery with no complications.

Learning point

Wounds of the heart by needles are rare injuries, but are serious $(1,2)$. Complications

may demand immediate surgical Intervention, such complications include tamponade, valvular dysfunction, arterial embolism and infection.

References

1. Potek IJ, Wright JS. Needle in the heart. Heart. 2007;45(3):325-7. 
2. Schechter D, Gilbert L. injuries of the heart and great vessels due to pins and needles. thorax.bmj.com. $1969 ; 24(2): 246-53$.
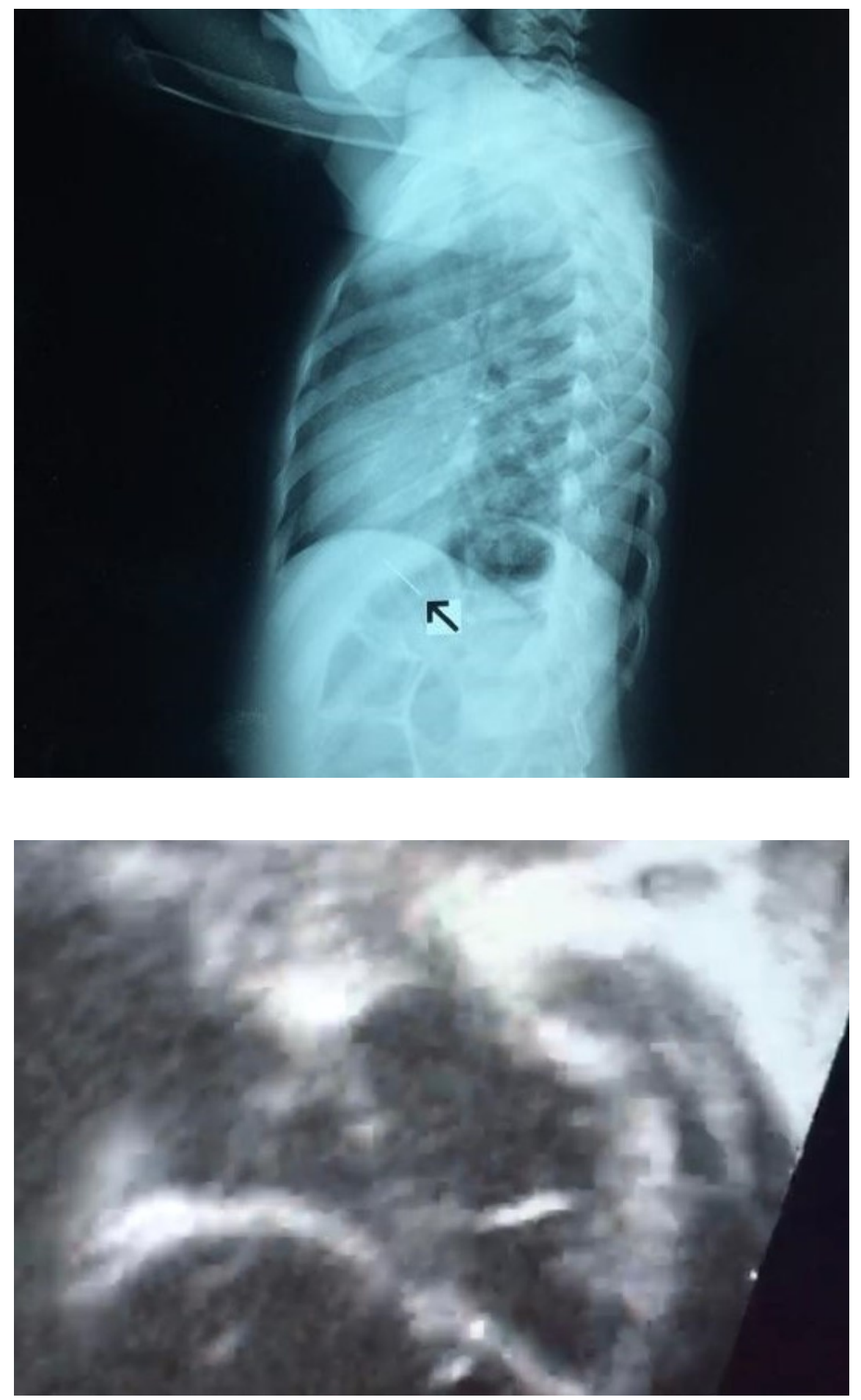